\title{
Complex rib anomalies in patient undergoing PET/CT study — a case report
}

Marcin Pachowicz¹, Grzegorz Staskiewicz², Grzegorz Opielak², Katarzyna Scibisz-Dziedzic¹, Beata Chrapko ${ }^{1}$

${ }^{1}$ Chair and Department of Nuclear Medicine, Medical University of Lublin, Poland

${ }^{2}$ Chair and Department of Human Anatomy, Medical University of Lublin, Poland

[Received 7 VI 2016; Accepted 9 I 2017]

A 51-year-old patient was referred to the Nuclear Medicine Department due to multifocal skin cancer. Four lesion were surgically removed. All of them were carcinoma basocellulare.

Routine ${ }^{18} \mathrm{~F}-\mathrm{FDG}-\mathrm{PET} / \mathrm{CT}$ scan was performed covering area from the base of the skull to the mid of the thigh. It began 60 minutes after radiotracer administration. No glucose avid areas suggestive of active neoplastic disease were observed (Figure 1).

The entire patient's chest was significantly malformed with rib abnormalities on both sides (Figures 2 and 3).

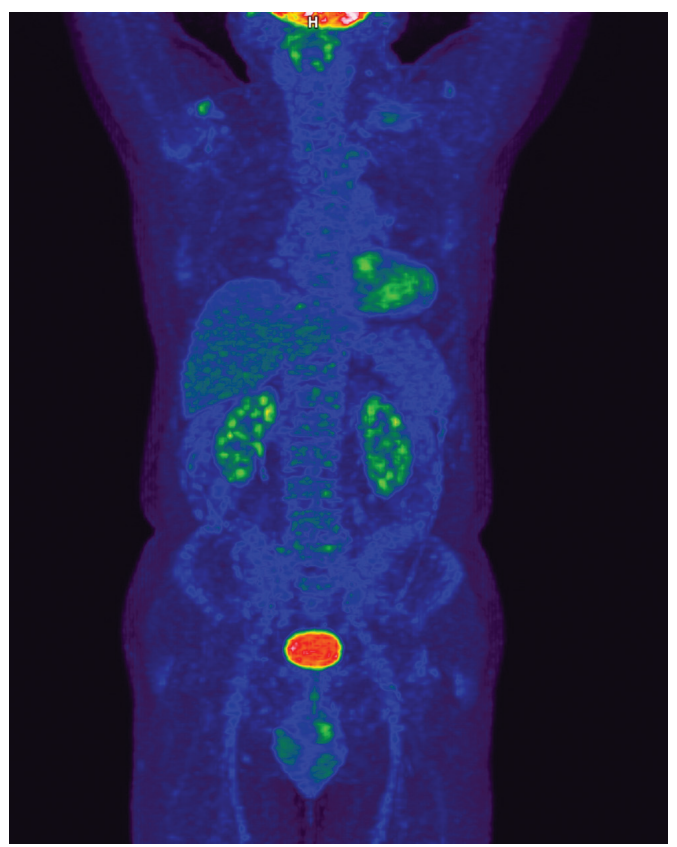

Figure 1. Maximum intensity projection ${ }^{18} \mathrm{~F}-\mathrm{FDG}-\mathrm{PET} / \mathrm{CT}$. No signs of metastases

Correspondence to: Marcin Pachowicz, MD Chair and Department of Nuclear Medicine. Medical University of Lublin

ul. Jaczewskiego 8c, 20-954 Lublin, Poland Tel/fax: + 48817244339

E-mail: marcin.pachowicz.md@gmail.com
Additionally there were cysts in the left maxilla and mandible (Figure 4).

The Gorlin-Goltz Syndrome (nevoid basal cell carcinoma syndrome, jaw cyst bifid rib syndrome) is an autosomal dominant inherited disorder. The incidence is estimated 1 in 50,000-150,000. It is characterized by multiple odontogenic keratocyst and basal cell carcinoma as well as skeletal malformations (hemivertebrae, scoliosis, syndactyly, polydactyly, shortened $4^{\text {th }}$ metacarpal bone, prognathism, calcification in the falx cerebri and tentorium cerebelli) and dental, ophthalmic and neurological abnormalities. Up to $60 \%$ of patients present rib anomalies. Moreover, these patients are more prone to multiple neoplasm such like medulloblastoma, meningioma, fibrosarcoma and cardiac fibroma, so early diagnosis is crucial in preventing complications. The first medical professional usually visited by the patients is the dentist. Multiple cystic lesions in the maxilla or mandible should be suggestive for odontogenic keratocyst and this syndrome.

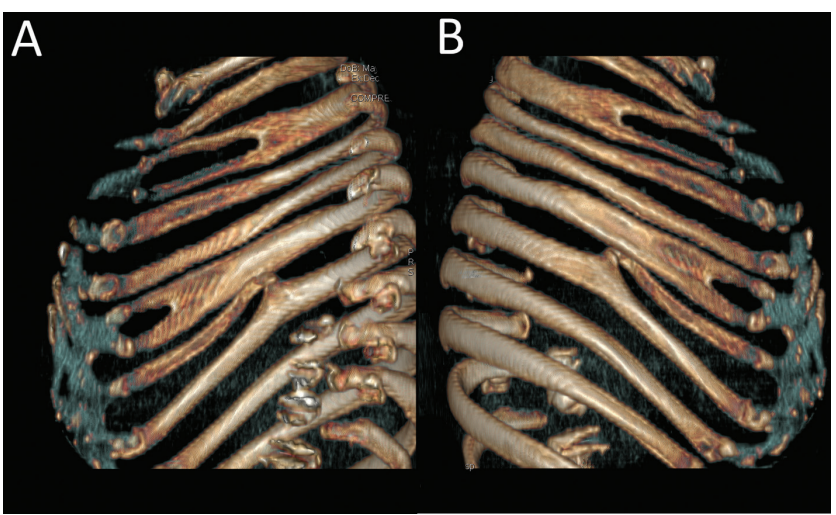

Figure 2. 3D volume rendering scans from $\mathrm{PET} / \mathrm{CT}$, right side of the patient chest (the view from outside and inside). The $3^{\text {rd }}$ rib bifurcated from the mid-axillary line to the costal cartilage, which reunites these ribs into one single sternal attachment. The $6^{\text {th }}$ rib similarly, but the bifurcation located more anteriorly (anterior axillary line) with one single cartilage. Additional rib arising from the $7^{\text {th }}$ one in the midaxillary line, then it runs a regular course being slightly depressed compared to other ribs. In the axial CT scans there was a space between these ribs. Costal cartilages were more separated in this case 


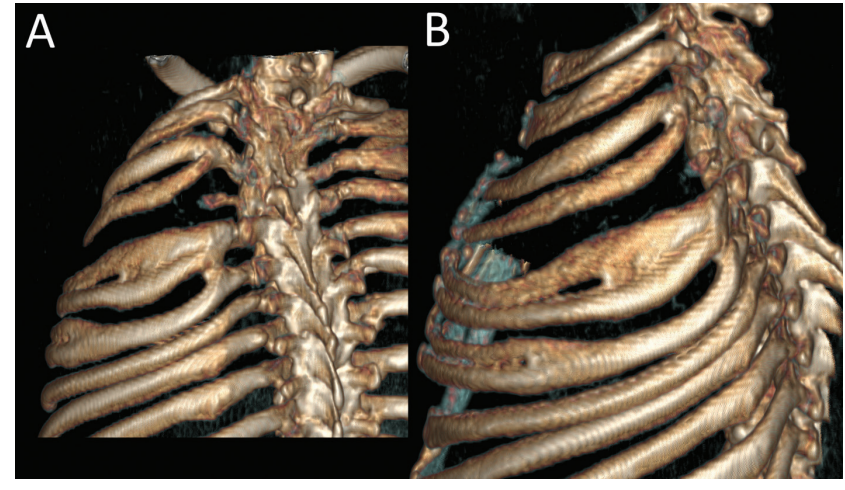

Figure 3. 3D volume rendering scans from PET/CT, left side of the patient chest (multiple views) The $3^{\text {rd }}$ rib bifurcated very close to the vertebral column (approximately $1 \mathrm{~cm}$ laterally from the tubercle). Both ribs run regular course and have separated costal cartilages and sternal attachments. The $4^{\text {th }}$ rib bridges with the $5^{\text {th }}$ one just next to the tubercles. Anteriorly both ribs bifurcate and both had separated costal cartilages. Between the $3^{\text {rd }}$ and $4^{\text {th }}$ ribs there is small rudimentary rib not articulating with vertebrae. Note also scoliosis with vertebral malformations

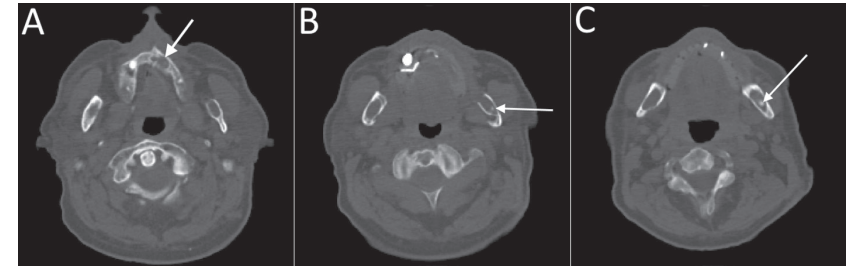

Figure 4. Left maxilla presents a cyst (about $1 \mathrm{~cm}$ ) in close relation to the incisive canal. Additionally two more cysts are found in the mandibular rami. In the left one there are two hyperdense areas inside, suggestive of unerupted teeth. Mandibular canals are incorporated into these cysts 